\title{
Efficacy of Telmisartan in Pristane Induced Arthritis Rat Model
}

${ }^{1}$ Quratulain Mehdi, ${ }^{2}$ Noaman Ishaq, ${ }^{3}$ Saba Batool, ${ }^{4}$ Kulsoom Farhat, ${ }^{5}$ Saman Omer, ${ }^{4}$ Arooj Shahid

${ }^{I}$ Department of Pharmacology, Quetta Institute of Medical Sciences, Quetta

${ }^{2}$ Department of Pharmacology, Bakhtawar Amin Medical and Dental College, Multan

${ }^{3}$ Department of Pharmacology, CMH Multan Institute of Medical Sciences, Multan

${ }^{4}$ Department of Pharmacology, Army Medical College, Rawalpindi

${ }^{5}$ Department of Pharmacology, Mohtarma Benazir Bhutto Shaheed Medical College, Mirpur AJK

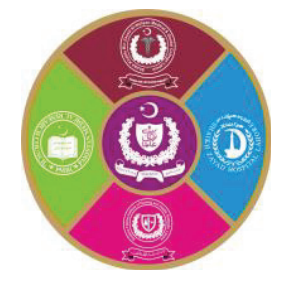

\begin{abstract}
Introduction: Rheumatoid arthritis is one of the most common systemic inflammatory diseases characterized by progressive damage to the joints. There is rising evidence that Renin Angiotensin Aldosterone System signaling is also involved in the inflammatory response in rheumatoid arthritis and its blockers possess anti-arthritic properties. Telmisartan is an angiotensin receptor blocker and PPAR- $\gamma$ agonist and its anti-arthritic effects were evaluated.

Aims \& Objectives: This experimental study was designed to evaluate the anti-arthritic efficacy of telmisartan in pristane induced rat model of arthritis in adult female rats.

Place and duration of study: The study was conducted in the Department of Pharmacology, Army Medical College, Rawalpindi, in collaboration with National Institute of Health and Armed Forces Institute of Pathology from July 2020 to August 2020.

Material \& Methods: Twenty four (24) adult non-pregnant female Sprague Dawley rats were divided in three groups $(\mathrm{n}=8)$ designated as Group A (normal control), Group B (arthritic control) and Group C (telmisartan group) \& maintained on standard diet and water adlibitum. Rheumatoid arthritis was induced in each rat of Groups B \&C by a single intradermal injection of $0.5 \mathrm{ml}$ pristane at the base of its tail on day 0 and the disease developed in two weeks. All 3 groups were given distilled water $2.5 \mathrm{ml} / \mathrm{kg}$ from 2-4 weeks and Group C was additionally given dissolved telmisartan orally at $2 \mathrm{mg} / \mathrm{kg} / \mathrm{day}$. Anti-arthritic efficacy was determined by assessing arthrogram score and total leukocyte count on day 0,14 and 28 along with histological examination done at the end of the study. Data analysis was done using SPSS version 25 .
\end{abstract}

Results: Healthy rats in group A maintained a unremarkable arthogram \& histogram score \& TLC count of $6675 \pm 350 / \mu 1$ during the entire study period. Telmisartan administration in Group $\mathrm{C}$ for two weeks after pristane induction resulted in significant reduction in arthrogram score (AS) $9.5 \pm 3.66$, total leukocyte count (TLC) $7350 \pm 550 / \mu 1$ and histological score (HS) to $6.88 \pm 1.24$ as compared to (AS) $14.50 \pm 2.07$, WBC $10150 \pm 350 / \mu \mathrm{L} \&$ (HS) $10.75 \pm 2.05$ in Group B, left untreated with pristane alone. The intergroup comparison showed significant $p$ value $<0.05$ respectively.

Conclusion: Anti-arthritic effect was shown by telmisartan as it was able to ameliorate the changes induced by pristane.

Key words: Anti-arthritic efficacy, Telmisartan, Pristane, Rheumatoid arthritis

\section{INTRODUCTION}

$\mathrm{R}_{\mathrm{h}}$ heumatoid arthritis (RA) is one of the most common inflammatory disorders occurring due to multiple factors including environmental and genetic risk factors. ${ }^{1}$ Clinical presentation of symmetrical joint involvement comprises of arthralgia, swelling, redness and limited mobility. The risk of development of extra articular manifestations such as keratitis, rheumatoid nodules, pericarditis and pleuritis is increased with poor control or severe disease. It is responsible for continuing disability, premature death and increased socioeconomic burden. ${ }^{2}$

Several strategies are employed in managing the disease. Surgical approaches help in improvement of functional activity and structural misalignment of joints but considered as a last resort. Physical and occupational therapy helps in relieving the muscular spasm and related joint stiffness. ${ }^{3}$ Current therapies include Non Steroidal Anti-Inflammatory Drugs (NSAIDS), Glucocorticoids (GCs) and biological agents which improve pain, fatigue and disability with more focus on controlling synovitis. ${ }^{4}$ Treatment modalities with fewer side effects are required for improvement of therapy. 
The Renin Angiotensin Aldosterone System (RAAS) is well known for its function as regulator of blood pressure but there is accumulating validation that its signaling is involved in reaction to inflammation identified in various disease states. Angiotensin II Type 1 Receptor (AT1R) can be presumed as a potential therapeutic target as rodent models of arthritis have massively expressed its presence in cultured RA-fibroblast like synoviocytes (FLS) as well as in hyperplastic synovium. The role of Angiotensin Converting Enzyme Inhibitors (ACEIs) and Angiotensin Receptor Blockers (ARBs) in ameliorating the laboratory and clinical parameters has also been documented. ${ }^{5}$ Telmisartan showed betterment in the parameters of arthritis in a rat model of RA. It exerts anti-inflammatory actions by restraining Tumor Necrosis Factor $\alpha$ (TNF- $\alpha)$ production and inhibiting pro-inflammatory mediators such as Interleukin-6 (IL-6) and Nuclear Factor kappa beta $(\mathrm{NF}-\kappa \beta){ }^{6}$

Various experiments and studies are being carried out to explore the anti-inflammatory properties of telmisartan with regards to various conditions. The current study is intended to demonstrate the antiarthritic effects of telmisartan in rat model of pristane induced arthritis (PIA).

\section{MATERIAL AND METHODS}

Study design: Laboratory based experimental study.

Ethical approval: The Ethics Review Committee of "Center for Research in Experimental and Applied Medicine (CREAM)", Army Medical College gave the approval for study.

Setting: It was carried out in department of Pharmacology and Therapeutics, Army Medical College (AMC), Rawalpindi in collaboration with National Institute of Health (NIH), Islamabad and Armed Forces Institute of Pathology (AFIP). Study was conducted for duration of one month from July to August 2020 and the animals were kept in animal house of NIH Islamabad.

Sampling Technique and size: Twenty Four (24) adult non-pregnant female Sprague Dawley rats weighing 250-300 grams were randomly divided into three groups of eight animals each.

Experimental setup: The rats were acclimatized for a week before the start of experiment. They were retained in wire topped cages and standard environmental conditions were maintained with temperature ranging between $25 \pm 5^{\circ} \mathrm{C}$ and $50 \pm 10 \%$ humidity. Free excess to clean drinking water and standard rodent diet ad libitum was provided during the study duration. Twenty four (24) adult non- pregnant female Sprague Dawley rats were divided in three groups $(\mathrm{n}=8)$ and were assigned as

Group A (normal control): Comprised of healthy rats. Intradermal injection of $0.1 \mathrm{ml}$ normal saline was given at the base of tail at day 0 and rats were given distilled water $2.5 \mathrm{ml} / \mathrm{kg} /$ day orally from 14 to 28 days.

Group B (arthritic control): Rheumatoid arthritis was induced with pristane on day 0 and rats were given distilled water $2.5 \mathrm{ml} / \mathrm{kg} /$ day orally from 14 to 28 days after induction.

Group C (telmisartan group): Rheumatoid arthritis was induced with pristane on day 0 and rats were treated with telmisartan manufactured by Pharm Evo (Pvt) Ltd in a dose of $2 \mathrm{mg} / \mathrm{kg} /$ day orally for two weeks after induction. ${ }^{7}$

Induction of rheumatoid arthritis: Induction of RA was done by a single intradermal injection of $0.5 \mathrm{ml}$ of pristane purchased from Martin Dow Marker Ltd at the base of tail of each rat in Group B and Group C. ${ }^{8}$ Arthritis developed gradually over a period of two weeks.

Assessment of arthritic progression by arthrogram score: The assessment of arthritic progression was done by clinical scoring of joint inflammation on all four limbs on alternate days from week 0 to 4 weeks after pristane injection. It ranged from $0-4$, where $0=$ no swelling and redness; $1=$ single joint involvement; $2=$ involvement of two joints; $3=$ involvement of more than two joints; $4=$ severe arthritis involving whole paw and digits. The collective minimum score for all four limbs would be 0 and maximum would be $16 .^{9}$ The arthrogram score at day 0,14 and 28 were statistically analyzed.

Hematological analysis: Blood was collected from the lateral tail vein on day 0,14 and 28 and was stored in Ethylene Diamine Tetra Acetic acid (EDTA) tubes for the analysis of Total Leukocyte Count (TLC). ${ }^{10}$ It was done by using Sysmex KX-21 automated analyzer.

Joint histopathology: All the rats were euthanized by chloroform at the end of the study on day 28 . The proximal part of tibia was sliced by using 5.5" angled bone cutter and right ankle joints were removed. Slides were prepared with Eosin and hematoxylin stains. Histological scoring was done according to a set criteria as described by Alabarse and his colleagues. For synovial inflammation, five high-power magnification fields were scored for the percentage of infiltrating mononuclear cells as follows: 0 , absent; 1 , mild $(1-10 \%)$; 2, moderate $(11-50 \%) ; 3$, severe $(51-100 \%)$; for synovial hyperplasia: 0, absent; 1, mild (5-10 layers); 2, moderate (11-50 layers); 3 , severe ( $>20$ layers); for 
extension of pannus formation: 0 , absent; 1 , mild; 2 , moderate; 3 , severe; for synovial fibrosis: 0 , absent; 1 , mild (1-10\%); 2, moderate (11-50\%); 3, severe $(51-100 \%)$; for cartilage erosion, that is, the percentage of the cartilage surface that was eroded: 0 , absent; 1 , mild (1-10\%); 2 , moderate (11-50\%); 3 , severe (51-100\%); and for bone erosion: 0 , none; 1 , minor erosion(s) observed only at high-power magnification fields; 2, moderate erosion(s) observed at low magnification; 3, severe transcortical erosion(s). ${ }^{11}$

\section{Statistical analysis:}

The Statistical Package for the Social Sciences (SPSS) version 25 was used for data analysis with quantitative data expressed as Mean \pm Standard Deviation (SD). One way of analysis of variance (ANOVA) was applied to observe the mean difference among control and experimental group and compared by Post Hoc Tukey's test. The difference between two observations was considered significant if the $\mathrm{p}$ value was equal to or less than $0.05(\mathrm{p} \leq 0.05)$.

\section{RESULTS}

The arthrogram score was zero in all the groups at day 0 . It remained zero in group A throughout the study duration whereas in group B it reached a mean of $13.5 \pm 2.97$ by day 14 and reached a mean of $14.5 \pm 2.07$ by day 28 . Fig- 1 shows a rat of group B with arthrogram score 12. Arthrogram score of group $C$ reached a mean of $12.5 \pm 3.96$ by day 14 and was declined after treatment with telmisartan by day 28 with a mean of $9.5 \pm 3.66$. Fig-2 shows a rat of group $\mathrm{C}$ with arthrogram score 04 .

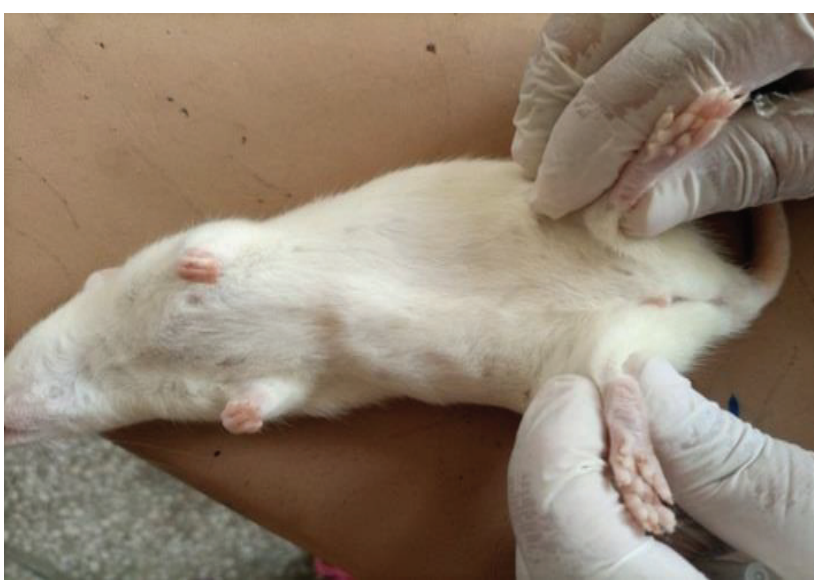

Fig-1: Rat of Group B with hind paws showing edema and redness

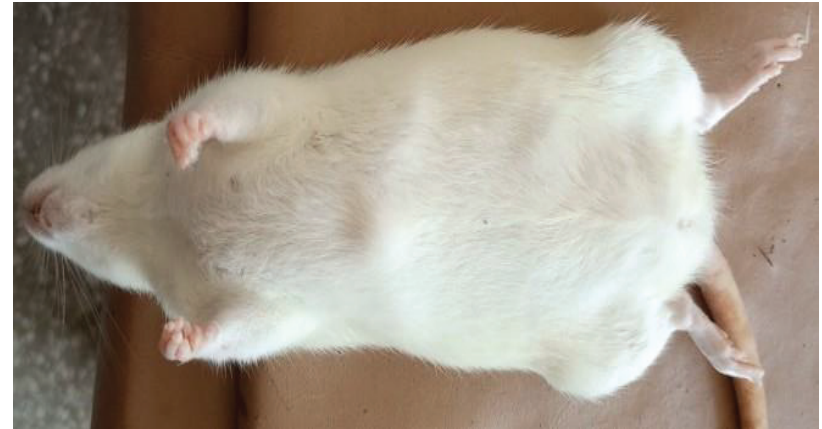

Fig-2: Rat of Group C with improved arthrogram score.

The mean TLC of group A, B and $\mathrm{C}$ was $6600 \pm 300 / \mu \mathrm{L}, \quad 6562 \pm 450 / \mu \mathrm{L}$ and $6488 \pm 360 / \mu \mathrm{L}$ respectively on day 0 . It remained almost constant in group A on day 14 and day 28 with a mean of $6600 \pm 310 / \mu \mathrm{L}$ and $6675 \pm 380 / \mu \mathrm{L}$. TLC was increased on day 14 in the other two groups with mean of $9988 \pm 510 / \mu \mathrm{L}$ and $9987 \pm 710 / \mu \mathrm{L}$. Group B was left untreated and its mean TLC on day 28 was $10150 \pm 350 / \mu \mathrm{L}$ whereas a dosing schedule of 2 $\mathrm{mg} / \mathrm{kg} /$ day of telmisartan led to a decrease in levels of TLC to $7350 \pm 550 / \mu \mathrm{L}$.

The histological examination of group A revealed minimum score as it was disease free and did not show any microscopic changes. The synovium lining was smooth with no penetration of infiltrating cells. Maximum score was seen in the rats of group $\mathrm{B}$ which ranged from 8-13. Marked percentage of infiltrating mononuclear cells was seen in four out of six slides while rest showed moderate infiltration. Two slides showed severe synovial hyperplasia but was moderate in other slides. The extension of pannus formation was maximum in two slides while the others showed mild to moderate pannus formation. Six out of eight slides showed moderate synovial hyperplasia. All the slides showed cartilage erosion. Mild to moderate presence of bone erosion was seen in all slides except one. Mean score of this group was $10.75 \pm 2.05$. Fig-3 shows a slide of Group $B$ with a score of 10 showing bone erosion and inflammation with infiltrating cells. Slides of group C showed improvement. The score ranged from 5-9. Two slides showed moderate infiltrating mononuclear cells, two showed no infiltration while four showed mild infiltration of mononuclear cells. Mild to moderate synovial hyperplasia was present in all the slides. Mild pannus formation and synovial fibrosis was a feature of all the groups. Five slides showed mild cartilage erosion, whereas the remaining slides showed moderate erosion. Mild bone erosion was seen in five slides and moderate in two slides. The mean score of this group was $6.88 \pm 1.24$. Fig-4 shows a score of 07 with surface corrugation. 


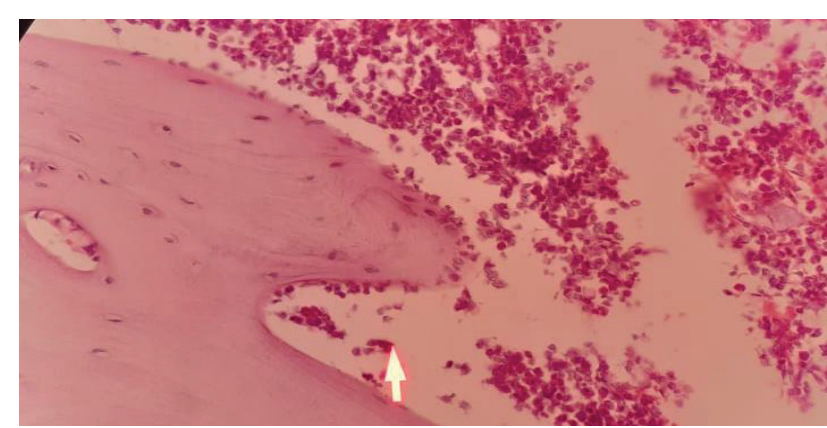

Fig-3: Photomicrograph of ankle joint of Group B

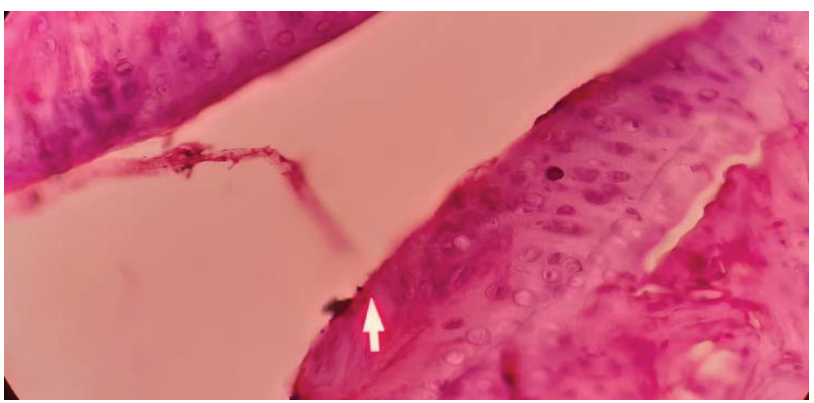

Fig-4: Photomicrograph of ankle joint of a rat of Group C

Treatment with telmisartan for two weeks decreased the arthrogram score, TLC and also showed improvement on histological examination. The intergroup comparison of the three groups of all the parameters depicted a statistically significant $p$ value $<0.001$ as shown in Table-1.

\begin{tabular}{|c|c|c|c|c|}
\hline Parameter & $\begin{array}{c}\text { Group A } \\
(\mathbf{n}=\mathbf{8})\end{array}$ & $\begin{array}{c}\text { Group B } \\
(\mathbf{n}=\mathbf{8})\end{array}$ & $\begin{array}{c}\text { Group C } \\
(\mathbf{n = 8})\end{array}$ & $\begin{array}{c}\text { p value } \\
\text { ANOVA }\end{array}$ \\
\cline { 2 - 3 } \pm SD & Mean \pm SD & Mean \pm SD & \\
\hline $\begin{array}{c}\text { Arthrogram } \\
\text { score }\end{array}$ & 0 & $14.50 \pm 2.07$ & $9.5 \pm 3.66$ & $<0.001 *$ \\
\hline $\begin{array}{c}\text { Total } \\
\text { Leukocyte } \\
\text { count (per } \boldsymbol{\mu L} \text { ) }\end{array}$ & $6675 \pm 380$ & $10150 \pm 350$ & $7350 \pm 550$ & $<0.001 *$ \\
\hline $\begin{array}{c}\text { Histopathology } \\
\text { score }\end{array}$ & 0 & $10.75 \pm 2.05$ & $6.88 \pm 1.24$ & $<0.001 *$ \\
\hline
\end{tabular}

Table-1: Intergroup comparison of arthrogram score, total leukocyte count and histopathology score on day 28.

\section{DISCUSSION}

RA is one of the common types of arthritis in the elderly population affecting the quality of life. The main pathological characters include persistent chronic synovitis, dysplasia of synovial membrane and pannus formation eventually causing cartilage and joint obliteration. ${ }^{12}$

PIA is one of the extensively used models for research purpose due to appreciative resemblance to the chemical characteristics of RA. ${ }^{13}$ It is similar to the condition seen in humans due to the presence of symmetrical joint involvement, presence of rheumatoid factor, bone and cartilage destruction as well as dependence of specific immunity seen in such models. ${ }^{14}$

In the present study the results indicated that the development of arthritis correlated with the increase in arthrogram score, TLC and histological score in Group B as compared to the Group A (normal control) till day 14. These findings are backed up by a number of studies conducted by researchers. Chen and his fellows in 2019 also observed the signs of severe arthritis spreading to ankle joints two weeks after injecting pristane. ${ }^{15}$ Regarding TLC, tissue homeostasis is maintained by the monitored movement of leukocytes between lymphoid and peripheral tissue through various stromal compartments. This trafficking is disturbed in RA. ${ }^{16}$ Feng and Qiu in 2018 successfully created a model of RA by subcutaneous injection of emulsion. The results indicated higher leukocyte count as well as damaged cartilage and considerable infiltration of inflammatory cells. ${ }^{17} \mathrm{We}$ followed a therapeutic model by commencing the treatment with telmisartan on day 14. It significantly lowered the aforementioned parameters when compared with the Group B (arthritic control).

Group C (telmisartan group) displayed the absence of swelling, redness and an overall decrease in the mean arthritic score. These findings were in accordance with the work of Hasanin and Mohamed who studied the anti-inflammatory effects of telmisartan in a rat model and saw a significant decrease in arthritic parameters. ${ }^{6}$ Two weeks treatment with telmisartan also displayed a decrease in TLC and histological score. Angiotensin II (Ang II) has a central role in leukocyte migration and infiltration at molecular and cellular level at the sites of inflammation. ${ }^{18}$ It is accountable for regulating proliferation of cells and apoptosis by binding to AT1R. Ang II exhibited from synovial tissue regulates synovial perfusion and growth by its actions on synovial AT1R. ${ }^{19}$ Thus the decrease in TLC can be justified by its AT1R blocking potential. Other than this, olmesartan, an ARB like telmisartan was used orally in a model of zymosan induced RA. It decreased the TLC with specific reduction in neutrophil count as well as showed improvement in histological score. ${ }^{20}$ This signifies that our experimental drug telmisartan may also have anti-arthritic effect against RA.

\section{Limitations of study:}

This study has also some limitations. Although the study showed that telmisartan has anti-arthritic properties, the preventive role of telmisartan should 
also be evaluated in such a model. More over future studies should also be conducted using this drug focusing on adverse effects

\section{CONCLUSION}

Oral administration of $2 \mathrm{mg} / \mathrm{kg} /$ day of telmisartan for two weeks significantly lowered the mean arthrogram score, total leukocyte count and histological score in a rat model of rheumatoid arthritis. Therefore, it can be concluded that telmisartan has anti-arthritic effects.

\section{Acknowledgement:}

Authors are thankful to Dr. Hussain Ali, Head of Research Animal Facility, National Institute of Health, Islamabad for his supervision regarding induction of Rheumatoid arthritis. A special thanks to Army Medical College, Rawalpindi for facilitating us to conduct this project.

\section{REFERENCES}

1. Abhishek A, Doherty M, Kuo CF, Mallen CD, Zhang W, Grainge MJ. Rheumatoid arthritis is getting less frequent-results of a nationwide population-based cohort study. Rheumatology. 2017 May 1; 56(5):736-44.

2. Guo Q, Wang Y, Xu D, Nossent J, Pavlos NJ, Xu J. Rheumatoid arthritis: pathological mechanisms and modern pharmacologic therapies. Bone Res. 2018 Apr 27; 6(1):1-4.

3. Bullock J, Rizvi SA, Saleh AM, Ahmed SS, Do DP, Ansari RA, Ahmed J. Rheumatoid arthritis: a brief overview of the treatment. Medical PrincPract. 2018; 27(6):501-7.

4. $\mathrm{Xu}$ Q, Zhou Y, Zhang R, Sun Z, Cheng LF. Antiarthritic activity of Qi-Wu rheumatism granule (a Chinese herbal compound) on complete Freund's adjuvant-induced arthritis in rats. Evid Based Complement Alternat Med. 2017 Jan 1; 2017:1960517

5. Terenzi R, Manetti M, Rosa I, Romano E, Galluccio F, Guiducci S, Ibba-Manneschi L, Matucci-Cerinic M. Angiotensin II type 2 receptor (AT2R) as a novel modulator of inflammation in rheumatoid arthritis synovium. Sci Rep. 2017 Oct 16; 7(1):1-1.

6. Hasanin AH, Mohamed RH. Telmisartan alone or in combination with etanercept improves anemia associated with rheumatoid arthritis in rats: a possible role of anti-inflammatory and renoprotective effects. Pharmacol Rep. 2020 Apr; 72(2):379-88.

7. Yanagihara H, Ushijima K, Arakawa Y, Aizawa KI, Fujimura A. Effects of telmisartan and olmesartan on insulin sensitivity and renal function in spontaneously hypertensive rats fed a high fat diet. J Pharmacol Sci. 2016 Jul 1; 131(3):190-7.
8. Faisal R, Gul M, Anwar A. Low doses of pristane in comparison with high doses in induction of arthritis in female-spraguedawley rats. Khyber Med Univ J. 2016 Dec 24; 8(4):185-8.

9. Gul A, Kunwar B, Mazhar M, Faizi S, Ahmed D, Shah MR, Simjee SU. Rutin and rutin-conjugated gold nanoparticles ameliorate collagen-induced arthritis in rats through inhibition of $\mathrm{NF}-\mathrm{\kappa B}$ and iNOS activation. IntImmunopharmacol. 2018 Jun; 1(59):310-7.

10. Faisal R, Chiragh S, Popalzai AJ, Rehman KU. Anti inflammatory effect of thymoquinone in comparison with methotrexate on pristane induced arthritis in rats. J Pak Med Assoc. 2015 May 1; 65(5):519-25.

11. Alabarse PV, Lora PS, Silva JM, Santo RC, Freitas EC, de Oliveira MS, Almeida AS, Immig M, Teixeira VO, Filippin LI, Xavier RM. Collagen-induced arthritis as an animal model of rheumatoid cachexia. Journal of cachexia, sarcopenia and muscle. 2018 Jun; 9(3):603-12.

12. Cai P, Jiang T, Li B, Qin X, Lu Z, Le Y, Shen C, Yang $\mathrm{Y}$, Zheng L, Zhao J. Comparison of rheumatoid arthritis (RA) and osteoarthritis (OA) based on microarray profiles of human joint fibroblast-like synoviocytes. Cell biochemistry and function. 2019 Jan; 37(1):31-41.

13. Wang B, Zhao P, Zhou Y, Meng L, Zhu W, Jiang C, Wang L, Cai Y, Lu S, Hou W. Increased expression of Th17 cytokines and interleukin-22 correlates with disease activity in pristane-induced arthritis in rats. Plos one. 2017 Nov 28; 12(11):e0188199.

14. Ruiz JT, Luján L, Blank M, Shoenfeld Y. Adjuvantsand vaccines-induced autoimmunity: animal models. Immunologic research. 2017 Feb 1; 65(1):55-65.

15. Chen Q, Zhang X, Xiong Y, Chen C, Lv S. The $\mathrm{CD} 25+/ \mathrm{CD} 4+\mathrm{T}$ cell ratio and levels of $\mathrm{CII}, \mathrm{CIX}$ and CXI antibodies in serum may serve as biomarkers of pristane-induced arthritis in rats and Rheumatoid Arthritis in humans. Comparative Biochemistry and Physiology Part C: Toxicology \& Pharmacology. 2019 Mar 1; 217:25-31.

16. Buckley CD, McGettrick HM. Leukocyte trafficking between stromal compartments: lessons from rheumatoid arthritis. Nature Reviews Rheumatology. 2018 Aug; 14(8):476-87.

17. Feng FB, Qiu HY. Effects of Artesunate on chondrocyte proliferation, apoptosis and autophagy through the PI3K/AKT/mTOR signaling pathway in rat models with rheumatoid arthritis. Biomedicine \& Pharmacotherapy. 2018 Jun 1; 102:1209-20.

18. Piqueras L, Sanz MJ. Angiotensin II and leukocyte trafficking: New insights for an old vascular mediator. Role of redox-signaling pathways. Free Radical Biology \& Medicine. 2020 Feb11:157:38-54

19. Wu Y, Lu X, Li M, Zeng J, Zeng J, Shen B, Zeng Y. Renin-angiotensin system in osteoarthritis: A new potential therapy. International immunopharmacology. 2019 Oct 1:75:105796.

20. Guerra GC, de Menezes MS, de Araújo AA, de AraújoJúnior RF, de Medeiros CA. Olmesartan 
prevented intra-articular inflammation induced by zymosan in rats. Biological and Pharmaceutical Bulletin. 2016 Nov 1; 39(11):1793-801.

\section{The Authors:}

Dr. Quratulain Mehdi

Demonstrator,

Department of Pharmacology,

Quetta Institute of Medical Sciences, Quetta.

Dr. Noaman Ishaq

Assistant Professor,

Department of Pharmacology,

Bakhtawar Amin Medical and Dental College,

Multan.

Dr. Saba Batool

Assistant Professor,

Department of Pharmacology,

CMH Multan Institute of Medical Sciences, Multan.
Dr. Kulsoom Farhat

Associate Professor,

Head, Department \& Pharmacology,

Army Medical College, Rawalpindi.

Dr. Saman Omer

Demonstrator,

Department of Pharmacology,

Mohtarma Benazir Bhutto Shaheed Medical

College, Mirpur AJK.

Dr. Arooj Shahid

Demonstrator,

Department of Pharmacology,

Army Medical College, Rawalpindi.

\section{Corresponding Author:}

Dr. Quratulain Mehdi

Demonstrator,

Department of Pharmacology,

Quetta Institute of Medical Sciences, Quetta.

E-mail: qurat.mehdi84@gmail.com 